\title{
Optimal agenda-setter timing
}

\author{
Mattias K. Polborn Department of Economics, \\ University of Illinois \\ Gerald Willmann Department of Economics, KU Leuven
}

Abstract. We analyze the optimal timing problem of an agenda setter who can propose a project that is then voted on by a committee. The payoff consists of a common stochastic component and an idiosyncratic component that differs among committee members. The agenda setter may be biased for or against the project, relative to the median committee member, and chooses when to call the vote. We analyze how the timing decision depends on the political environment and the bias of the agenda setter. We show that both positively and negatively biased agenda setters can implement decisions that differ from those preferred by the median committee member. JEL classification: C7, D7

Le timing optimal du maître de l'ordre du jour. On analyse le timing optimal d'un maitre de l'ordre du jour qui peut proposer un projet sur lequel un comité va alors se prononcer. Le gain a deux composantes : l'une commune et stochastique, et l'autre idiosyncratique et variable selon les membres du comité. Le maître de l'ordre du jour peut pencher en faveur ou contre le projet, par rapport au membre médian du comité, et il choisit le moment du vote. On analyse comment cette décision du moment du vote dépend de l'environnement politique et du biais du maître de l'ordre du jour. On montre que les maîtres de l'ordre du jour (en faveur ou contre le projet) peuvent engendrer des décisions qui diffèrent de celles préférées par le membre médian du comité.

\section{Introduction}

We consider the following, common public decision problem: A committee has to decide whether and when to implement a public project with known costs, but

We are grateful to Dan Bernhardt and Klaus Schmidt, as well as to two anonymous referees for valuable comments and suggestions. This paper was written while Willmann was visiting the Department of Economics at the University of Illinois, which he thanks for its hospitality. The usual caveat applies: all remaining errors are ours. Email: gerald.willmann@econ.kuleuven.be 
uncertain benefits. If the project is implemented, each voter has to pay a cost and then starts to receive a payoff stream composed of an idiosyncratic and a common stochastic component. The former captures the notion that voters differ in their preferences towards the project. The common component of the payoffs follows a Brownian motion, which implies that waiting with the decision to implement the project yields additional valuable information and generates an option value of waiting, as in the theory of real investment options. ${ }^{1}$ As in the case of investment problems, this setup leads to an optimal threshold for each voter that is higher than the point where she just breaks even in expectation.

The difference between a firm's investment problem and the committee's problem is that the payoffs of the project are different for different members, so that some members want to implement the project sooner than others. We analyze the optimal use of proposer power by an agenda setter who is biased relative to the median committee member and can decide when to call a vote. Upon the agenda setter's proposal, the committee votes and the project is implemented or not. The optimal timing decision depends on the political environment and the bias of the agenda setter.

There are many important applications of our model of irreversible political decisions. One example is the question of whether to join a free trade area or a common currency like the Euro, or the dissolution of nation states, as in Quebec's 1995 independence referendum. Each of these projects could be implemented immediately, or voters could wait to learn more about the potential payoffs before deciding; of course, the opportunity cost of the latter course of action is that no payoffs accrue during the waiting time. Similarly, many internal political projects have the property that the decision on whether to implement is discrete and difficult to reverse, and that payoffs are heterogeneous among agents, uncertain, and develop over time: for example, think of the decision on whether to develop a new type of fighter jet or to build large infrastructure projects like the Strait of Messina bridge in Italy or the Oresund crossing between Denmark and Sweden.

In all of these examples, the key elements of our model are present. First, waiting to decide allows agents to learn more about the systematic component of payoff risk. Second, preference divergence between the agenda setter and the median voter is a realistic assumption in many applications. In Quebec, for example, the decision on whether to call a referendum on the question of independence from Canada was made by the premier of Quebec at the time, Jacques Parizeau, the leader of the strongly pro-independence Bloc Québécois. Obviously, since the independence referendum failed, the median voter opposed independence at the time. However, even among those who preferred separation from Canada, some voters' most preferred course of action might have been to wait longer and see how matters developed for Quebec within Canada.

We assume that, once the agenda setter proposes implementation, it is up to the committee to decide by majority vote. Clearly, the agenda setter's right to

1 See Dixit and Pindyck (1994) for a review of the literature on investment under uncertainty. 
decide on timing gives real power to an agenda setter who is negatively biased relative to the median voter, because she can simply delay the vote until even she herself regards the project as worthwhile. The case of a positively biased agenda setter is more interesting. At first sight, it may seem as if she has no particular power, because she cannot enforce the implementation of a project as long as the median committee member opposes it. However, upon closer inspection this argument misses one crucial aspect. While it is indeed the case that the status quo can be changed only if the median member approves the project, the agenda setter, by scheduling a vote now, can effectively eliminate the option value of waiting for the median committee member. As long as the median member breaks even in expectation without the option value, she will vote in favour of the project, even though she would have preferred to wait longer before making the decision. The power of the agenda setter thus stems from her ability to force committee members to forgo the option value of waiting by scheduling a vote now. Our model therefore shows that the prerogative of the agenda setter to decide on timing endows her with considerable power.

This is also evidenced by more recent events in Canada. Fulfilling a campaign pledge, Prime Minister Stephen Harper introduced a law (Bill C-16) in 2006 requiring that each general election take place on the third Monday of October in the fourth calendar year after the previous poll. He pitched this legislation as a means of stopping prime ministers from calling snap votes whenever the political tide felt favourable, only to subsequently circumvent the spirit of the law by calling an early election in 2008. Clearly, breaking the spirit of his own law was politically costly; hence, there must have been a substantial benefit to calling an early election.

We also show that the resulting welfare loss for the median committee member can be diminished or avoided if a suitable supermajority rule is used when deciding on project implementation. Many constitutions require that changes to them need some supermajority to pass, and many clubs require a supermajority to accept new members. To the extent that many of these settings are also characterized by uncertainty and learning opportunities, our model provides a new justification for such rules.

In an extension of our model, we analyze the effects that arise when the agenda setter does not know the exact preference parameter of the median voter. This mitigates a positively biased agenda setter's desire to hold the vote early. An important implication of this case is that the proposal can actually fail, as proposals sometimes do in the real world. We also consider another source of uncertainty, namely, the risk for the agenda setter of losing her gatekeeper position. This type of uncertainty induces a positively biased agenda setter to call a vote earlier than in the basic case. Taken together, both types of uncertainty increase the probability that proposals fail, which enhances the empirical appeal of our model.

Our paper is related to different strands of literature. Most important, the current paper is related to an important literature in political science and political economy that explores the sources of power of an individual dealing with a 
committee. That power may arise for two reasons. First, as explored in the literature on vote buying (see, e.g., Dal Bo 2007), the agenda setter may be able to offer incentives to the members of the group and possibly play them against each other. Another reason is that the individual may have the ability to manipulate the agenda. The latter literature has emphasized the role played by status quo positions, open vs. closed rules, and supermajority requirements. Our paper is the first to show that the option value of waiting also creates agenda-setting power.

The literature on agenda setting power was pioneered by the seminal contribution of Romer and Rosenthal (1978). This model has become one of the most widely applied models in political science to describe the effects of legislative organization. For a review of the literature on legislative organization and committee power, see Krehbiel (2004). In models of proposer power with horizontally differentiated preferences, the power of the proposer depends on the relative positions of the status quo and on the median voter's and the proposer's bliss points. We analyze similar questions, though for binary decisions, but in a dynamic setting that gives rise to learning opportunities.

As for dynamic aspects, Smith $(1996,2003)$ analyzes the decision of the government in parliamentary democracies such as Britain or Canada on whether to call an early election. In his model, the government has more information on its ability or the economic development in the next time period. The government is inclined to call an early election if it wants to pre-empt the revelation of unfavourable information. However, rational voters take early elections as a negative signal and become more suspicious of the government. While our model has uncertainty about future payoffs, information is symmetric, so that no suspicion effect arises when the agenda setter moves. By focusing on the dynamic aspect, the current paper contributes to the nascent literature on dynamic political economy and its efficiency properties. Besley and Coate (1998) generalize the citizen candidate model to a two-period setting and show that equilibrium policies are often not Pareto efficient in a dynamic setting, because a Pareto-improving policy may affect voters' future preferences and thus the identity of the future policy maker in a way that is unfavourable for the present office holder. While this effect is absent in our basic model and equilibrium policies are always Pareto efficient in our framework, they are skewed towards the preferences of the agenda setter and do not maximize a median voter's utility.

Eraslan and Merlo (2002) analyze a bargaining model in which at least $q$ out of $n$ players need to agree for a proposal to be implemented and the size of the surplus and the identity of the proposer change stochastically between periods. As in our paper, excessively early implementation may arise in equilibrium, but for different reasons. In their model, players who receive a positive share in the current proposal fear that they could be excluded from the implementation coalition in future periods if the present proposal fails. This fear may induce them to agree to implementation proposals before the efficient time, and, in turn, the proposer makes an acceptable implementation proposal earlier than optimal, because approval by the necessary $n-1$ other players is relatively cheap and 
there is a risk that the identity of the proposer changes in the next period. In contrast, the 'sharing rule' is given exogenously in our paper, as there are no transfers between players, but rather the implementation payoff of each player depends on his preference type (with a fixed sharing rule, the equilibrium in Eraslan and Merlo 2002 is efficient). Also, in our model, the proposer's power depends crucially on her ability to commit to not repeating a proposal, which kills the median's option value of waiting. In contrast, no such commitment is feasible in Eraslan and Merlo (2002).

Meredith (2008) analyzes a model of strategic timing of referenda by agendasetters, in which concurrent elections affect the composition of the electorate. An agenda setter with power over the timing of a referendum can schedule the referendum either in conjunction with a general election (so that the turnout is high) or as a stand-alone referendum (with lower turnout), depending on which electorate is more likely to approve his proposal. In our model, such an effect does not arise, as the agenda setter always faces the same committee composition.

An important effect in our model is the option value of waiting, which draws on the literature on investment under uncertainty and real options (e.g., McDonald and Siegel 1986; Abel and Eberly 1994; see Dixit and Pindyck 1994 for an introduction). The main difference between that literature and our model is that, in investment problems, a single decision maker aims to maximize profit. In our model, committee members and agenda setter have different preferences over the implementation of the project and hence about the optimal duration of learning. Moreover, the agenda setter is in a position to manipulate voters' option value of waiting through her choice of timing. There also exists, of course, a large literature on each of the different projects that we envision - for example, the formation, size, and dissolution of political or economic unions - and on large infrastructure projects. Our model complements this literature by analyzing the political timing decision explicitly.

The remainder of the paper is organized as follows. The next section presents the basic version of our model. In section 3 , we analyze the decision of an individual committee member, subsequently focus on the proposer's problem, and offer a discussion of the results. Section 4 considers the two extensions of the model, and the last section concludes.

\section{The model}

A committee consisting of a finite set of members has the option to implement a project. As explained in the introduction, the project can be thought of as entering the European Union, adopting the Euro, or implementing a large infrastructure project. If the project is undertaken at time $t$, it generates a flow net payoff of $v(\tau)+b_{i}$ for each member $i$ of the committee at all times $\tau>t$. We refer to $v(\tau)$ as the common payoff (equal for all members), while $b_{i}$ is member $i$ 's idiosyncratic bias towards the project. Without loss of generality, we normalize the median 
value of $b$ in the committee to 0 . Committee members with above-median benefits have $b>0$, while those with below-average benefits have $b<0$. Undertaking the project requires an investment of $C$ by every committee member.

At any time $t$, committee members know the present instantaneous payoff $v(t)$, but not how $v$ will develop in the future. Rather, the future development of $v$ follows a Brownian motion without drift:

$$
d v=\gamma d z
$$

Here, $d v$ is the change of $v$ over a short time interval, $d z$ is the increment of a standard Wiener process, and the coefficient $\gamma$ determines the variance of the process, that is, measures the amount of uncertainty about the future payoffs of the project. ${ }^{2}$ The assumption that $v$ changes randomly over time is made to capture the notion that future payoffs are uncertain, but correlated over time. For example, if adopting the Euro gives a high average payoff today, it will likely also give a high payoff tomorrow. For analytical convenience, we assume that there is no deterministic drift in $v$, but such a generalization would be straightforward.

Once the project is implemented, it generates payoffs for all following time periods; that is, the project implementation is irreversible. ${ }^{3}$ Hence, if the project is implemented at time $t$, committee member $i$ 's expected net benefit is

$$
U_{i}=E\left(\int_{t}^{\infty}\left[v(\tau)+b_{i}\right] \delta^{(\tau-t)} d \tau\right)-C,
$$

where $\delta$ is the discount factor. In the following, it is sometimes useful to work with the instantaneous interest rate $\rho=\ln (1 / \delta)$, where $\rho$ is defined such that $\delta^{d t}=$ $1-\rho d t$ for small intervals $d t$.

The political decision process is as follows. The decision to implement the project is taken by majority vote in the committee where each member faces a binary decision (yes or no). ${ }^{4}$

2 A Wiener process has the property that its increment after $t$ units of time is a normally distributed random variable with expected value 0 and variance $t$; it can be interpreted as the continuous time limit of a random walk. The parameter $\gamma$ just multiplies the standard increment, so that the variance of $v$ in $t$ periods is $\gamma^{2} t$. See, for instance, Dixit and Pindyck (1994) for further details.

3 If project abandonment were possible, the results would not change qualitatively. Dixit (1989) allows for possible 'exit' in an investment context.

4 Hence, we let the committee decide solely on the implementation of the project and exclude proposals that also directly redistribute money between committee members. In order to focus on the power of the agenda setter that derives from her ability to manipulate the timing of the agenda, it is useful to disregard another potential source of agenda-setter power that derives from a monopoly power to propose side-payments between voters. 


\section{Results}

\subsection{The individuals' problem}

As a preliminary step, suppose that a type $b$ individual could individually choose the value threshold $v_{b}^{*}$, such that the project is implemented as soon as $v \geq v_{b}^{*}$. This is essentially an investment problem, where the option value of waiting must be considered. Note that $v_{b}^{*}$ is time independent, as the future development of the payoffs depends only on the current value of $v$. Because the random process has no drift, so that $E(v(\tau))=v(t)$ for all $\tau \geq t$, type $b$ receives the following expected discounted payoff after implementation:

$$
\frac{v(t)+b}{\ln (1 / \delta)}-C=\frac{v(t)+b}{\rho}-C .
$$

To determine $v_{b}^{*}$, it is helpful to analyze the problem for some general implementation threshold $\tilde{v}$. Let $F_{b}(v, \tilde{v})$ denote the expected discounted continuation utility if today's state is $v$ and investment is made when, for the first time, $v(t)=\tilde{v}>v$. At date $t+d t$, the value of $v$ will change, so $F_{b}(\cdot, \tilde{v})$ must satisfy the following equation for small $d t$ :

$$
F_{b}(v, \tilde{v})=\delta^{d t} E\left(F_{b}(v(t+d t), \tilde{v})\right)=(1-\rho d t) E\left(F_{b}(v(t+d t), \tilde{v})\right)
$$

for all $v(t)<\tilde{v}$, that is, in the waiting interval. To calculate $E\left(F_{b}(v(t+d t), \tilde{v})\right)$, we can take a Taylor expansion, where we suppress the time argument of $v(t)$ and let the prime denote the derivative with respect to the first argument. ${ }^{5}$

$$
E\left(F_{b}(v(t+d t), \tilde{v})=F_{b}(v, \tilde{v})+F_{b}^{\prime}(v, \tilde{v}) E(d v)+\frac{1}{2} F_{b}^{\prime \prime}(v, \tilde{v}) E\left(d v^{2}\right) .\right.
$$

From (1), we know that $E(d v)=\gamma E(d z)=0$ and $E\left(d v^{2}\right)=\gamma^{2} E\left(d z^{2}\right)=\gamma^{2} d t$. Using this information in (4) and rearranging, we get

$$
\rho F_{b}(v, \tilde{v})=\frac{1}{2} \gamma^{2} F_{b}^{\prime \prime}(v, \tilde{v}) .
$$

One boundary condition for this differential equation follows from the fact that, as $v$ grows very negative, it becomes extremely improbable that the project can be implemented soon and payoffs that accrue only far in the future are discounted heavily. Hence, for any given $\tilde{v}$, it must be true that $\lim _{v \rightarrow-\infty} F_{b}(v, \tilde{v})=0$. Taking this boundary condition into account, the solution of (6) is

$$
F_{b}(v, \tilde{v})=K_{b}(\tilde{v}) \exp \left(\frac{\sqrt{2 \rho}}{\gamma} v\right),
$$

5 Note that, by Ito's Lemma (see Dixit and Pindyck 1994), the variance of $v$ is linear in $d t$, and therefore the last term on the right-hand side of (5) cannot be discarded even in a first-order Taylor expansion with respect to $d t$. 
where $K_{b}$ depends on the implementation threshold $\tilde{v}$ and is pinned down by the following value-matching condition that, as $v$ approaches the investment threshold $\tilde{v}$, the continuation value $F$ must approach the implementation payoff (3):

$$
F_{b}(\tilde{v}, \tilde{v})=\frac{\tilde{v}+b}{\rho}-C .
$$

We can now solve for the optimal implementation threshold $v_{b}^{*}$ by maximizing the right-hand side of (7) over $\tilde{v}$. Using (8) in (7), solving for $K_{b}(\tilde{v})$ and differentiating yield the following 'smooth pasting' condition:

$$
K_{b}\left(v_{b}^{*}\right) \frac{\sqrt{2 \rho}}{\gamma} \exp \left(\frac{\sqrt{2 \rho}}{\gamma} v_{b}^{*}\right)=\frac{1}{\rho} .
$$

Using (7), (8), and (9) to solve for $v_{b}^{*}$, we obtain

$$
v_{b}^{*}=\rho C+\frac{\gamma}{\sqrt{2 \rho}}-b .
$$

At the individually optimal threshold, the payoff $v_{b}^{*}+b$ is equal to the annuitized cost $\rho C$ plus the term $\gamma / \sqrt{2 \rho}$, which can be interpreted as the benefit of waiting. The more uncertain is the future development of $v$ (i.e., the larger is $\gamma$ ) and the lower the time preference (i.e., the lower is $\rho$ ), the larger is the benefit of waiting. For future reference, we note that

$$
F_{b}\left(v, v_{b}^{*}\right)=\frac{\gamma}{\rho \sqrt{2 \rho}} \exp \left(\frac{\sqrt{2 \rho}}{\gamma}\left(v-v_{b}^{*}\right)\right) .
$$

Before turning our attention to the agenda setter's problem, it is useful to summarize graphically the results derived so far. Figure 1 depicts the continuation value $F_{b}\left(v, v_{b}^{*}\right)$ for the optimal implementation threshold as well as the payoff from implementation, $\rho C+\gamma / \sqrt{2 \rho}-b . F_{b}(\cdot)$ satisfies both value matching and smooth pasting with the implementation payoff $\left(v^{*}+b\right) / \rho-C$, as one flows smoothly into the other at $v_{b}^{*}$. A higher idiosyncratic bias $b$ shifts both $F_{b}$ and the implementation payoff to the left and therefore leads to a lower individually optimal implementation threshold. Without an option value of waiting (i.e., if $\gamma / \sqrt{2 \rho}=0$ ), the investment threshold would be at $\rho C-b$ where the expected net payoff is zero.

\subsection{The proposer's problem}

We now turn to the problem of the agenda setter. It is easy to see that, if $b_{p}<0$ that is - if the agenda setter is more critical of the project than the median committee member, the former will call a vote exactly when $v(t)$ reaches $v_{b p}^{*}$, as given by (10). The agenda setter is unconstrained by the requirement that a majority vote in favour of the project. Since she is more critical of the proposal 


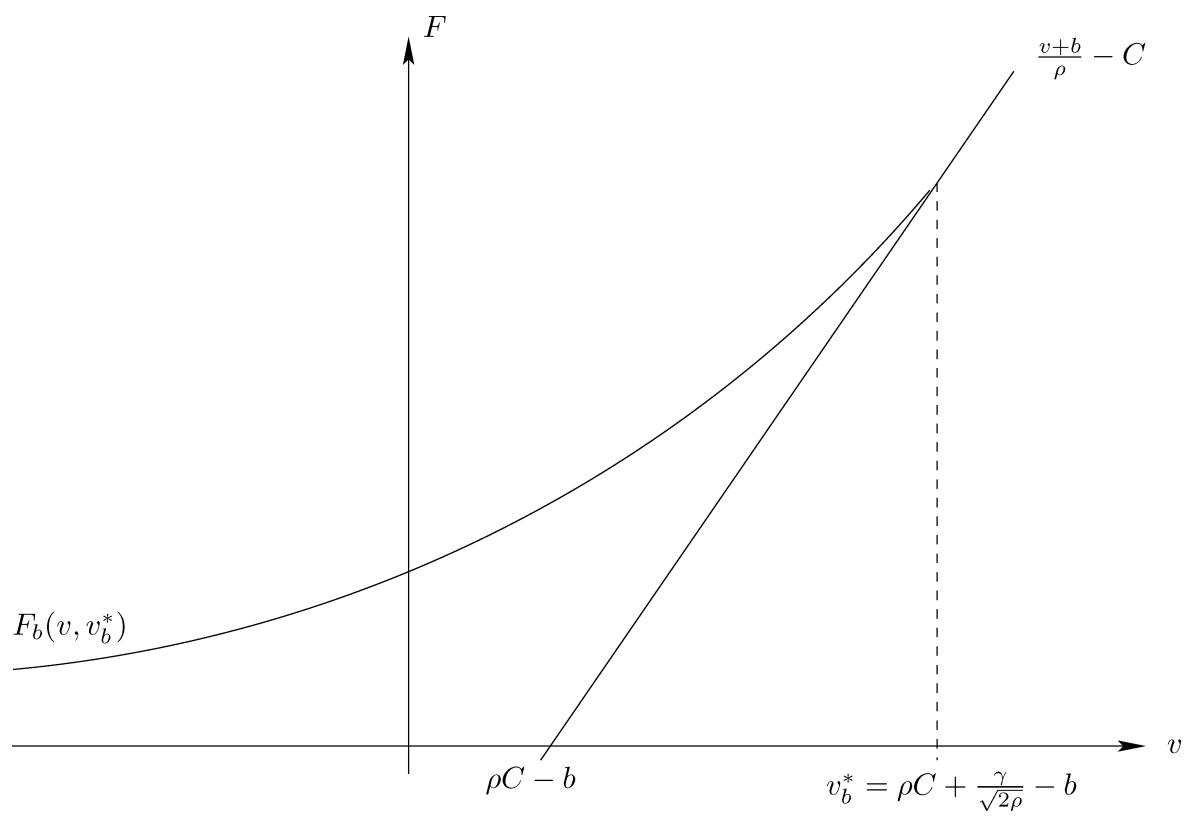

FIGURE 1 Continuation and implementation value

than the median committee member, the majority of the committee certainly thinks that the project should be implemented when (even) the proposer finds that the project is worthwhile.

The more interesting case is where the agenda setter has a positive bias relative to the median $\left(b_{p}>0\right)$. We assume that the agenda setter commits not to call another vote, should the first one fail. Note that the assumption that the proposer can commit is a standard assumption in the literature on agenda-setter power. We discuss this assumption and possible alternatives in greater detail below.

Consider the subgame that follows after the proposer calls a vote. Committee members vote in favour of the implementation of the project if and only if their net benefit as given by (3) is positive. The indifferent member is characterized by $b^{\text {ind }}=\rho C-v(t)$. The proposer needs to ensure that $b^{\text {ind }} \leq 0$; otherwise the proposal will fail and the project will never be implemented. We can call $v(t) \geq$ $\rho C$ the proposer's majority implementation constraint.

Suppose first that $b_{p} \in[0, \gamma / \sqrt{2 \rho}]$; that is, the proposer's bias in favour of the project is smaller than the benefit of learning. Equation (10) then implies that

$$
v_{b_{p}}^{*}=\rho C+\frac{\gamma}{\sqrt{2 \rho}}-b_{p} \geq \rho C,
$$

so that, in this case, the agenda setter is unconstrained by the majority implementation constraint and calls the vote as soon as $v(t) \geq v_{b_{p}}^{*}$. 
To see this graphically, suppose the curve depicted in figure 1 is the one of the median committee member with $b=0$. By calling the vote, the agenda setter forces the median member to forgo the option value of waiting and to make a decision based solely on the expected discounted payoff. That is, the threshold for the median is now the horizontal intercept of the implementation payoff at $\rho C$. The agenda setter's curve (not shown) resembles the curve of the median member, but is shifted to the left by $b_{p}$. Since this shift to the left falls short of the option value of waiting when $b_{p}<\gamma / \sqrt{2 \rho}$, the optimal threshold of the agenda setter $v_{b_{p}}^{*}$ lies to the right of the decision threshold of the median member at $\rho C$. The approval of the median member is therefore not a binding constraint, because whenever $v(t) \geq v_{b_{p}}^{*}$, it also exceeds $\rho C$. In other words, as long as $b_{p}<\gamma / \sqrt{2 \rho}$, the agenda setter calls the vote such that there is a supermajority of committee members approving the proposal.

If $b_{p}>\gamma / \sqrt{2 \rho}$, so that that the agenda setter's bias exceeds the option value of waiting, then the proposer's (unconstrained) preferred threshold $b_{p}^{*}$ exceeds the decision threshold of the median member. The need to win approval of a majority of the committee thus poses a binding constraint, and the agenda setter calls the vote only when $v(t) \geq \rho C$, that is, as soon as the median member's net benefit becomes non-negative. We collect our findings in the following proposition:

Proposition 1. A proposer with $b_{p} \leq \gamma / \sqrt{2 \rho}$ calls the vote as soon as $v(t) \geq v_{b_{p}}^{*}$. A proposer with $b_{p} \geq \gamma / \sqrt{2 \rho}$ does so as soon as $v(t) \geq \rho C$. If $b_{p}>0$, the project is implemented earlier than is optimal for the median committee member.

\subsection{Discussion}

\subsubsection{Option value of learning and proposer power}

The source of proposer power for a positively biased agenda setter is the option value of learning. As a consequence of the agenda setter's ability to destroy this option value, a supermajority of the committee supporting the implementation of a project does not necessarily imply that the project should have been implemented earlier and that the agenda setter has a negative bias relative to the median committee member. Only the most extreme agenda setters (those with $b_{p}>\gamma / \sqrt{2 \rho}$ ) are constrained by the majority implementation constraint, while all other agenda-setter types receive a supermajority for their proposal.

If $\gamma$ is zero, so that payoffs do not change over time, then a positively biased proposer must implement the median's preferred threshold $v_{0}^{*}=\rho C$, because the majority implementation constraint requires $v(t) \geq \rho C$ at the threshold. In this case, a positively biased proposer can successfully implement the project only if the median member benefits from the project (and therefore also wants to implement it right away). In contrast, the larger is $\gamma$ (and hence the option value of waiting), the larger is the range of biased agenda setters who are unconstrained by the majority implementation constraint. 


\subsubsection{Commitment}

If the agenda setter has a positive bias, the ability to make a take-it-or-leave-it offer to committee members is decisive for her power to implement a policy that is not the one preferred by the median committee member. This mirrors the results in static models on proposer power in open-rule versus closed rule legislatures. Our model corresponds to a closed rule legislature, where the proposal by the committee in charge of a particular piece of legislation (i.e., the agenda setter in our terminology) cannot be amended by the parent legislature, but rather receives an up-or-down vote. In the static horizontal-differentiation case, a (say) right-biased committee has the power to implement a policy different from the median's bliss point both when the status quo is to the left and when it is to the right of the median. Likewise, the agenda setter in our model can implement a different investment time than that preferred by the median, whether the agenda setter is positively or negatively biased.

In contrast, under an open rule, the median of the legislature can amend a proposal such that it conforms with its ideal point (see Denzau and Mackay 1983). A right-biased committee in the standard model will therefore table legislation only if the status quo is to the left of the median, or if the status quo is so far to the right that the committee prefers the median's ideal point to the status quo. Similarly, if the proposer in our model could not commit at all not to call a new vote after a possible defeat of the project, then the median committee member would reject any premature implementation and be sure that it would be ex post optimal for the proposer to reschedule a vote at any time when the median voter was willing to support the project (and hence at the median voter's preferred time).

In practice, while the agenda setter may not be able to commit to never calling a vote again, reputational effects are likely to make it credible to wait at least for a fixed time $T$ before rescheduling a vote. In this case, the longer is $T$, the more of the median's option value the proposer can destroy by scheduling a vote and, consequently, the less restrictive is the majority implementation constraint.

Formally, consider the problem of the median committee member when deciding whether to vote for or against a proposal. Implementation yields an expected payoff of

$$
E U_{I}(v(t))=\frac{v(t)}{\rho}-C
$$

for the median committee member. In contrast, after a rejection, the project is implemented when, after waiting at least a time $T, v$ surpasses $\hat{v}$ for the first time. Let $\phi\left(v ; v(t), \gamma^{2} T\right)$ denote the probability density function of random variable that is normally distributed with expected value $v(t)$ and variance $\gamma^{2} T$ (i.e., this is the pdf of the value of $v$ after $T$ periods have passed). The median's expected payoff after a rejection is 


$$
\begin{aligned}
& E U_{R}(v(t)) \\
& \quad=e^{-\rho T}\left[\int_{-\infty}^{\hat{v}} F_{b}(v, \hat{v}) \phi\left(v ; v(t), \gamma^{2} T\right) d v \int_{\hat{v}}^{\infty}\left(\frac{v}{\rho}-C\right) \phi\left(v ; v(t), \gamma^{2} T\right) d v\right] .
\end{aligned}
$$

The second integral consists of those realizations of $v$ that lead to immediate implementation after waiting $T$ periods, while the first integral consists of those realizations of $v$ that are below the implementation threshold after $T$ periods. In both cases, these (expected) payoffs accrue only after $T$ periods, so that they need to be discounted by $e^{-\rho T}$.

The median voter will vote for implementation if and only if $E U_{I}(v(t)) \geq$ $E U_{R}(v(t))$, and thus the critical value $\hat{v}$ is determined by $E U_{I}(\hat{v})=E U_{R}(\hat{v})$. Clearly, as $T$ goes to infinity, $\lim _{T \rightarrow \infty} E U_{R}=0$. Thus, the implementation constraint goes to the same value as in the basic model above $(\hat{v}=\rho C)$. In contrast, if commitment is possible for only a finite length of time $T$, the median voter will agree only to more valuable projects, and if $T$ is sufficiently short, the implementation constraint becomes binding for the agenda setter.

The observation that a reputation to commit helps the proposer may explain the following interesting behaviour after failed proposals. For example, consider the 1996 Quebec independence referendum that failed very narrowly (49.4\% supported independence). It would appear to be at least conceivable that there could be a majority for independence if the referendum had been repeated after a short time, and since the Bloc Québécois remained in power in the provincial government of Quebec until 2003, they could have scheduled a new referendum any time, but chose not to.

Our results suggest that committing to not repeating a vote after a potential failure is crucial for the proposer, as it destroys the value of waiting for the median committee member and may induce her to agree to the proposer's project before the median's optimal threshold is reached. In other words, if the Bloc had called a referendum too soon after the first one, it would not only have destroyed this reputation. Also, if voters do not believe that the vote is final, then some moderate independence supporters may choose not to support immediate independence, as they prefer to wait longer. Hence, if the Bloc had called a new referendum, it would probably have failed by a larger margin.

\subsubsection{Welfare}

When the proposer is biased relative to the median voter, she will implement the project too early or too late from the median's point of view. (Provided that the median is close to the average, this also means that the implementation timing is suboptimal from a utilitarian welfare perspective) ${ }^{6}$ For a positively biased

6 Note that Pareto optimality has no bite as a welfare criterion, because any relevant implementation threshold is necessarily Pareto optimal: if the agenda setter can implement her 


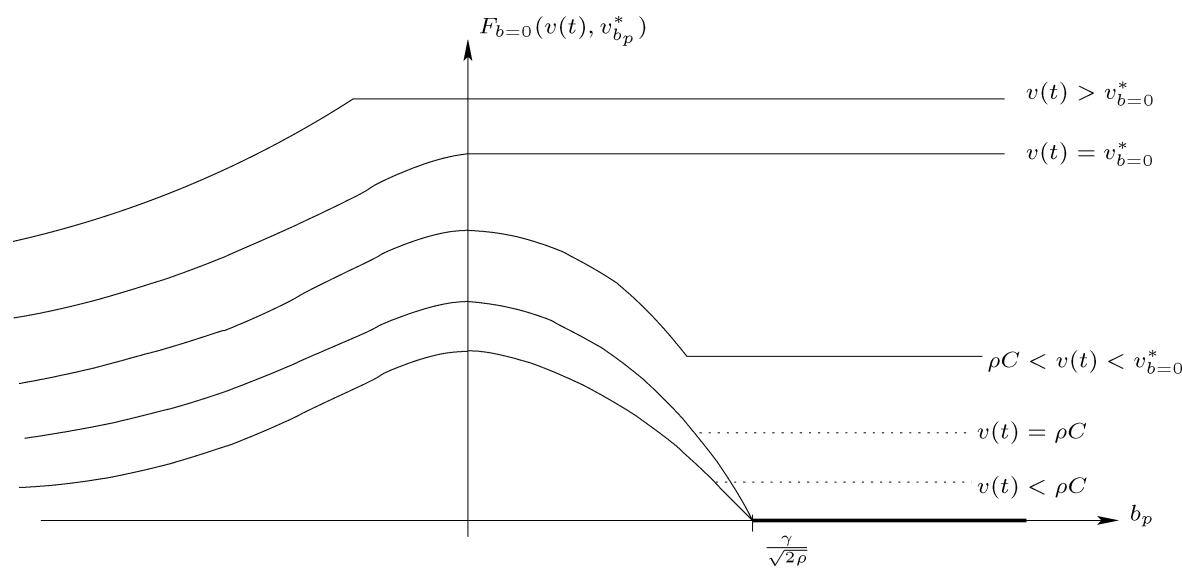

FIGURE 2 Median value as function of proposer's bias

proposer, this inefficient outcome arises because the proposer forces the median to forgo the option value of waiting and achieves an outcome that is closer to her own preferences. ${ }^{7}$

Figure 2 addresses how the median committee member's utility depends on the bias of the agenda setter. Different curves in this figure correspond to different initial values of $v(t)$. Clearly, $b_{p}=0$ maximizes the median's utility for any initial value of $v(t)$, as the agenda setter then implements the median member's optimum.

Now consider the effect of proposer bias. If $v(t)$ exceeds or equals the threshold of the median member (the top two curves), she is well served by a positively biased agenda setter because both of them favour immediate implementation. In contrast, utility decreases if the proposer is (sufficiently) negatively biased.

The third curve corresponds to the case that $v(t) \in\left(\rho C, v_{b=0}^{*}\right)$. A positively biased agenda setter implements the project before the median's preferred time, destroying some of the option value of waiting. All types to the right of the kink in the third curve implement immediately, so that the median (as well as all other voters) is indifferent between these proposer types.

The bottom two curves correspond to the case that the current value of the project for the median is negative, so that the project is not implemented immediately. In this case, a sufficiently positively biased agenda setter will implement

optimal threshold without being constrained, then any change would decrease the agenda setter's utility; if the agenda setter is constrained by the majority implementation constraint, then the median member's preferred threshold exceeds the equilibrium threshold, while the agenda setter's preferred threshold lies at a lower value of $v$; clearly, no Pareto improvement is feasible.

7 As in the static literature on proposer power, we do not model the question of why the proposer is biased relative to the median committee member. For an empirical analysis of whether congressional committees are biased relative to their parent body, see, for example, Krehbiel (1990), Londregan and Snyder (1994), and Groseclose (1994). 
the project as soon as $v(t)=\rho C$, which destroys the entire option value of waiting. Therefore, a sufficiently positively biased agenda setter is potentially a lot worse in this case than any negatively biased one. (A negatively biased proposer implements too late, but when she does, the median has a strictly positive utility from the project. Hence, the expected discounted utility with a negatively biased proposer is always positive.)

\subsubsection{Optimal majority rules}

So far, we have assumed that the support that a project must achieve in order to be implemented is a simple majority. However, from an ex ante perspective of institutional design, it may be possible to choose a different majority rule to diminish the ability of the agenda setter to exploit the median voter.

Consider what would happen if the decision on whether to implement the project when a vote is called is made not by the median $b=0$, but rather by type $\tilde{b}=-\gamma / \sqrt{2 \rho}$, that is, a voter whose negative bias just equals the value of learning and therefore is just indifferent to implementing the project at the value threshold that characterizes the median's optimal implementation time. This transfer of power creates a new implementation constraint that is binding for all positively biased agenda setters, while it does not affect a negatively biased proposer, because that proposer type implements only projects that are even better. Hence, such a transfer of power to type $\tilde{b}=-\gamma / \sqrt{2 \rho}$ in the implementation vote is even guaranteed to be beneficial if the median voter does not know the type of proposer ex ante.

To choose a majority rule in order to make type $\tilde{b}$ pivotal in the implementation vote, note that when type $\tilde{b}$ is just indifferent towards the implementation of the project, then every type with $b>\tilde{b}$ strictly prefers to implement the project. Therefore, to make type $\tilde{b}$ pivotal for the decision, the required majority for a proposal to pass should be $1-\Phi(\tilde{b})=1-\Phi(-\gamma / \sqrt{2 \rho})$, where $\Phi(\cdot)$ is the cumulative distribution function of preference types in the committee. Note that $1-\Phi(-\gamma / \sqrt{2 \rho})>1 / 2$, as $\Phi(-\gamma / \sqrt{2 \rho})<\Phi(0)=1 / 2$, so the optimal majority rule is a supermajority rule.

This result provides some theoretical justification for legislation like the 'Clarity Act' passed by the Canadian Parliament after the failed Quebec referendum: a future independence referendum would require a 'clear' majority (as opposed to just a simple majority) in order for independence to go forward. ${ }^{8}$ More generally, many constitutions require that changes to them need some supermajority to pass, and many clubs require a supermajority to accept new members. To the extent that many of these settings are also characterized by uncertainty and learning opportunities, our model provides a justification of these supermajority requirements.

8 While the act does not specify an explicit supermajority, Prime Minister Jean Chrétien and his Intergovernmental Affairs minister, Stéphane Dion, frequently suggested that $60 \%$ would be a reasonable benchmark. 
The result that it may be optimal in a dynamic setting for the median voter to transfer power to some other voter is similar to Messner and Polborn (2004). ${ }^{9}$ They also study a dynamic setting in which the median voter of the initial election, through the adoption of a supermajority rule, aims to transfer power to another individual in future elections, as he expects that this individual will represent the present median voter's preferences better than the future median. In contrast to our model, the reason is that the median voter in Messner and Polborn (2004) expects that his tastes change in the future, while no such preference development is necessary to justify adoption of a supermajority rule in the present setting.

\section{Extensions}

We now analyze two extensions of the basic model. First, we consider the case where the exact preference of the median committee member is unknown, so that there is a risk for proposals to fail. ${ }^{10}$ Second, we consider what happens when there is the risk for the agenda setter of losing her proposer status, and then we combine both forms of uncertainty.

\subsection{Uncertainty about the median}

In contrast to the basic model, suppose now that the agenda setter is uncertain about the exact preferences of the median committee member and believes that the median's preference is distributed according to the cumulative distribution function $\Psi(\cdot)$. Since a vote called at value $v$ will succeed if and only if $\left(v+b_{M}\right) /$ $\rho-C \geq 0$, the probability of project implementation at $v$ is $1-\Psi(\rho C-v)<1$. It is convenient to denote $1-\Psi(\rho C-v)$ by $P(v)$; note that the probability $P(\cdot)$ is an increasing function of $v$. Let $H(v, \tilde{v})$ denote the continuation value of the agenda setter if her implementation threshold is $\tilde{v}$. In analogy to the basic model, this value function satisfies the following differential equation:

$$
\rho H(v, \tilde{v})=\frac{1}{2} \gamma^{2} H^{\prime \prime}(v, \tilde{v}) .
$$

Using $\lim _{v \rightarrow-\infty} H(v, \tilde{v})=0$ as boundary condition, we get $H(v, \tilde{v})=$ $K(\tilde{v}) \exp (\sqrt{2 \rho} v / \gamma)$, where $K(\tilde{v})$ is defined by the following value-matching condition:

9 Other papers in which the initial median voter may choose a supermajority rule to transfer power and for commitment purposes include Barbera and Jackson (2004) and Dal Bo (2006).

10 Another possibility for analyzing electoral uncertainty is to introduce a delay between the time when the vote is scheduled and when it is actually held. A sufficiently large decrease of $v$ between these times may lead to a failure of a scheduled vote. Since the algebra is considerably more complicated in this scenario and results are likely to be qualitatively similar, we focus on uncertainty about the median's position. 


$$
H(\tilde{v}, \tilde{v})=P(\tilde{v})\left(\frac{\tilde{v}+b_{p}}{\rho}-C\right) .
$$

The RHS of (16) represents the expected payoff from calling the vote at $v(t)=$ $\tilde{v}$, that is, the probability that it is successful times the expected payoff after implementation. The proposer chooses $\tilde{v}$ to maximize $H(v, \tilde{v})$, subject to (16). The first-order condition is

$$
\frac{\sqrt{2 \rho}}{\gamma} P(\tilde{v})\left(\frac{\tilde{v}+b_{p}}{\rho}-C-\frac{\gamma}{\rho \sqrt{2 \rho}}\right)=P^{\prime}(\tilde{v})\left(\frac{\tilde{v}+b_{p}}{\rho}-C\right) .
$$

The RHS of this condition is positive, as $P^{\prime}(\tilde{v})$ is positive and the agenda setter's payoff at the optimal threshold is positive. Hence, the LHS is also positive, so that the optimal threshold $v^{*}$ exceeds $\rho C+\gamma / \sqrt{2 \rho}-b_{p}$, the optimal threshold in the basic case. Thus, uncertainty about the median voter's preferences mitigates the result that a positively biased proposer implements the project too early:

PROPOSITION 2. If the median committee member's location is uncertain, then the agenda setter's optimal threshold exceeds the one in the baseline case.

\subsection{Timing with risk of losing the proposer status}

We now return to the assumption that the median's position is known, but assume that the agenda setter may eventually lose the power to schedule a vote. In particular, the loss of power is modelled as a Poisson process with arrival rate $\lambda$. If the proposer loses her position, she is replaced by the median committee member, who then schedules the vote according to our analysis in the basic model.

Consider first a proposer who is negatively biased against the project. It is quite clear that any type $b_{p}<0$ behaves exactly as in the basic model: when $v$ reaches the value threshold from the model without loss of status possibility, it is clearly optimal for the agenda setter to call a vote, rather than wait further. If $v(t)<v^{*}\left(b_{p}\right)$ and the agenda setter loses power, the project is implemented too early (from her point of view), but calling a vote early does not help against this problem.

For the rest of this section, we therefore consider the more interesting case of a positively biased agenda setter. The utility of the agenda setter if she loses her gatekeeper function without having called a vote is given by $F_{b_{p}}\left(v, v_{b=0}^{*}\right)$ for all $v \leq v_{b=0}^{*}$. We denote her value function as long as she is the proposer by $G(v, \tilde{v}, \lambda)$, where $\tilde{v}$ is the (not necessarily optimal) threshold at which she calls a vote. In what follows, we suppress the latter two arguments of $G(\cdot)$ when no confusion arises. For values of $v$, for which the agenda setter prefers to wait, the following condition holds:

$$
G(v)=(1-\rho d t)\left[(1-\lambda d t) E(G(v(t+d t)))+\lambda d t E\left(F_{b_{p}}\left(v(t+d t), v_{b=0}^{*}\right)\right)\right] .
$$

The right-hand side represents the discounted value of the agenda setter's expected payoff at $t+d t$ : either she keeps proposer status (with probability 
$1-\lambda d t)$ and receives $G(v(t+d t))$, or, with probability $\lambda d t$, she loses that power and receives the continuation utility $F_{b_{p}}\left(v(t+d t), v_{b=0}^{*}\right)$. Letting $d t$ go to zero and neglecting all terms of order higher than 1 in $d t$ yields the following differential equation:

$$
(\rho+\lambda) G(v)=\frac{1}{2} \gamma^{2} G^{\prime \prime}(v)+\lambda F_{b_{p}}\left(v, v_{b=0}^{*}\right) .
$$

This differential equation has the general solution

$$
G(v, \tilde{v}, \lambda)=K_{1} e^{\frac{\sqrt{2(\rho+\lambda)}}{\gamma} v}+K_{2} e^{-\frac{\sqrt{2(\rho+\lambda)}}{\gamma} v}+F_{b_{p}}\left(v, v_{b=0}^{*}\right),
$$

where $F_{b_{p}}$ is the value function from the baseline case defined in section $3, K_{2}$ must be zero, since $\lim _{v \rightarrow-\infty} G(v, \tilde{v}, \lambda)=0$ for any $\tilde{v}$ and $\lambda$, and $K_{1}$ must be positive, as $G\left(v, v_{b_{p}}^{*}, 0\right)=F_{b_{p}}\left(v, v_{b_{p}}^{*}\right)>F_{b_{p}}\left(v, v_{b=0}^{*}\right)$.

Proposition 3. Suppose that the agenda setter can lose her power with rate $\lambda$. Then, the agenda setter's optimal (unconstrained) implementation threshold decreases in $\lambda$.

Proof. Since value matching must hold at the implementation threshold, it is sufficient to show that $G(v, \tilde{v}, \lambda)$ is decreasing in $\lambda$. Consider an increase in $\lambda$, say, from $\lambda_{1}$ to $\lambda_{2}>\lambda_{1}$.

$$
\begin{aligned}
G\left(\tilde{v}, \tilde{v}, \lambda_{1}\right) & =G\left(\tilde{v}, \tilde{v}, \lambda_{2}\right) \text { implies } \\
K_{1}\left(\lambda_{1}\right) & =K_{1}\left(\lambda_{2}\right) \exp \left(\left[\frac{\sqrt{2\left(\rho+\lambda_{2}\right)}}{\gamma}-\frac{\sqrt{2\left(\rho+\lambda_{1}\right)}}{\gamma}\right] \tilde{v}\right),
\end{aligned}
$$

and we have

$$
\begin{aligned}
G\left(v, \tilde{v}, \lambda_{1}\right)-G\left(v, \tilde{v}, \lambda_{2}\right)= & K_{1}\left(\lambda_{2}\right) \exp \left(\frac{\sqrt{2\left(\rho+\lambda_{2}\right)}}{\gamma} \tilde{v}\right) \\
& \times\left[\exp \left(\frac{\sqrt{2\left(\rho+\lambda_{1}\right)}}{\gamma}(v-\tilde{v})\right)\right. \\
& \left.-\exp \left(\frac{\sqrt{2\left(\rho+\lambda_{2}\right)}}{\gamma}(v-\tilde{v})\right)\right]>0
\end{aligned}
$$

for all $v<\tilde{v}$.

Proposition 3 states that the higher the risk of losing her status, the earlier a positively biased agenda setter calls the vote. The intuition for this result is clear, because the value of waiting is diminished by the risk of losing power. ${ }^{11}$

11 The effect that the fear of losing power induces the proposer to act faster is also present in Eraslan and Merlo (2002). In their paper, this effect is the only motivation for a proposer to act 'too early,' while in our paper, it adds on the fact that an agenda setter who is certain to remain in power already implements too early. 
In particular, since the basic model corresponds to the special case of $\lambda=0$, the agenda setter's optimal threshold is lower than in the basic model.

Note that the agenda setter is still possibly constrained by the fact that she needs a majority; that is, $v^{*} \geq \rho C$. As $\lambda$ increases, the (unconstrained) optimal threshold for the agenda setter decreases, so that for increased $\lambda$ more types of agenda setter become constrained and will call the vote the first time it will result in a bare majority. If a type $b$ agenda setter is already constrained for some $\lambda=\lambda_{0}$, then further increases in $\lambda$ to $\lambda_{1}>\lambda_{0}$ do not decrease the threshold any further.

So far, we have assumed that, if the agenda setter is replaced, then the new proposer has the median's preferences. Alternatively, the new gatekeeper could be randomly drawn from the distribution of types. As argued above, the possibility of being replaced by an even more positively biased agenda setter would not lead to a decrease of the implementation threshold. However, since it is also possible to be replaced by a less positively or negatively biased proposer, the implementation threshold is still lower than in the case where the agenda setter retains her power with certainty.

A comparison between the cases when the replacement is the median and when the replacement is drawn from the distribution, with respect to the effect on the agenda setter's implementation threshold, is difficult to make and likely depends on the specific type distribution. For example, if the agenda setter is close to the median committee member, then being replaced by the median certainly is not a big hazard and hence will not decrease the implementation threshold significantly, relative to the basic model. However, if the replacement is instead drawn from the distribution, the replacement could be very negatively biased, and, to avoid this scenario, the agenda setter may be willing to lower the implementation threshold significantly.

If, instead, the discount rate and the agenda setter's positive bias is large, then being replaced by the median is almost as bad for the agenda setter as being replaced by a very negatively biased successor, as both will wait a long time until they implement. Therefore, the initial proposer may prefer a random draw from the distribution (which, after all, could also bring a positively biased replacement) to getting the median for sure, and if this is the case, the initial agenda setter's implementation threshold may well be higher for the random draw case than for the the median replacement with certainty case.

\subsection{Both types of uncertainty combined}

What happens if the agenda setter risks losing her status and does not exactly know the median? To understand this case, we have to combine the results from the two subsections. Analytically, this means combining equation (20) and the value-matching condition (16). Although no closed-form solution exists, it is clear that the combined effect on the optimal threshold is ambiguous: the risk of losing the status reduces the threshold, whereas the uncertainty about the median increases it. 
Despite this ambiguity, we can deduce one further interesting insight. Start with uncertainty about the median. As we saw above, under this scenario votes are lost with probability $1-P\left(v^{*}\right)>0$. Now, suppose that, in addition, the agenda setter faces the risk of losing her status. As we showed above, this tends to reduce her optimal threshold. As $v^{*}$ decreases and $P($.$) is increasing, the probability that$ a vote is lost increases. Hence, the larger the political uncertainty for the agenda setter's position, the higher the probability that a vote called by the agenda setter is lost.

\section{Conclusion}

In this paper, we have developed a model of agenda-setter timing that has a wide variety of applications, in particular to fundamental political changes such as entry into the European Union or into a currency union, or a declaration of independence. In these situations, learning about payoffs from (not) undertaking the project is often an important effect that makes it optimal to delay decisions, as is true for investment under uncertainty. A positively biased agenda setter can force the median voter to implement the project earlier than would be optimal for the median voter by destroying the option value of waiting. The agenda setter's power to implement a project before the time that is optimal for the median voter is larger, the more important learning is and the longer the politician can commit to not holding a second vote after a possible failure. If institutions can be designed to counter the proposer's power, it is optimal for the median voter to employ a supermajority rule for the implementation vote.

\section{References}

Abel, A., and J. Eberly (1994) 'A unified model of investment under uncertainty,' American Economic Review 84, 1369-84

Barbera, S., and M. O. Jackson (2004) 'Choosing how to choose: self-stable majority rules and constitutions,' Quarterly Journal of Economics 119, 1011-48

Besley, Timothy, and Stephen Coate (1998) 'Sources of inefficiency in a representative democracy: a dynamic analysis,' American Economic Review 88, 139-56

Dal Bo, Ernesto (2006) 'Committees with supermajority voting yield commitment with flexibility,' Journal of Public Economics 90, 573-99

- (2007) 'Bribing voters,' American Journal of Political Science 51, 789-803

Denzau, Arthur T., and Robert J. Mackay (1983) 'Gatekeeping and monopoly power of committees: an analysis of sophisticated and sincere behaviour,' American Journal of Political Science 27, 740-61

Dixit, Avinash (1989) 'Entry and exit decisions under uncertainty,' Journal of Political Economy 97, 620-38

Dixit, Avinash, and R. Pindyck (1994) Investment under Uncertainty (Princeton: Princeton University Press)

Eraslan, Hülya, and Antonio Merlo (2002) 'Majority rule in a stochastic model of bargaining,' Journal of Economic Theory 103, 31-48 
Groseclose, Timothy (1994) 'Testing committee composition hypothesis for the United States Congress,' Journal of Politics 56, 440-58

Krehbiel, K. (1990) 'Are congressional committees composed of preference outliers?' American Political Science Review 84, 149-63

- (2004) 'Legislative organization,' Journal of Economic Perspectives 18, 113-28

Londregan, J., and J. Snyder (1994) 'Comparing committee and floor preferences,' Legislative Studies Quarterly 19, 233-66

McDonald, R., and D. Siegel (1986) 'The value of waiting to invest,' Quarterly Journal of Economics 101, 707-28

Meredith, Marc (2008) 'The strategic timing of direct democracy,' mimeo, MIT

Messner, Matthias, and Mattias K. Polborn (2004) 'Voting on majority rules,' Review of Economic Studies 71, 115-32

Romer, T., and H. Rosenthal (1978) 'Political resource allocation, controlled agendas and the status quo,' Public Choice 33, 27-43

Smith, Alastair (1996) 'Endogenous election timing in majoritarian parliamentary systems,' Economics and Politics 8, 85-110

- (2003) Election Timing (Cambridge: Cambridge University Press) 